\title{
Existence and Stability of Anti-Periodic Solutions for Fuzzy Cohen- Grossberg Neural Networks with Time-varying Delays on Time Scales
}

\author{
Qianhong Zhang ${ }^{1, a, *}$, Guiying Wang ${ }^{1, b}$ and Xiaoying Zhong ${ }^{2, c}$ \\ ${ }^{1}$ School of Mathematics and Statistics, Guizhou University of Finance and Economics, Huaxi \\ Univerisity City, Guiyang, China \\ ${ }^{2}$ Library, Guizhou University of Finance and Economics, Huaxi Univerisity City, \\ Guiyang, China \\ azqianhong68@163.com, b1433287871@qq.com, czhongxiaoying801@163.com \\ ${ }^{*}$ corresponding author
}

Keywords: anti-periodic solutions, fuzzy Cohen-Grossberge neural networks, time-varying delays, asymptotic stability, time scales.

\begin{abstract}
By applying the novel method, some sufficient conditions are established for the existence and global exponential stability of anti-periodic solutions for a kind of fuzzy Cohen-Grossberg neural networks on time scales. Moreover an example is given to illustrate our results.
\end{abstract}

\section{Introduction}

Over the past few years, Cohen and Grossberg neural networks (CGNNs) [1] have been extensively studied and applied in many different fields such as associative memory, signal processing and some optimization problems. In such applications, it is of prime importance to ensure that the designed neural networks are stable [2]. In practice, due to the finite speeds of the switching and transmission of signals, time delays do exist in a working network and thus should be incorporated into the model equation. The dynamical behaviors, such as, the existence and stability of equilibrium point, periodic and almost periodic solutions, for CGNNs have been investigated for the sake of theoretical interest as well as application considerations.(see for example Refs. [3-10] and the references therein).

In this paper, we would like to integrate fuzzy operations into Cohen-Grossberg neural networks. Speaking of fuzzy operations, Yang and Yang [13] first introduced fuzzy cellular neural networks (FCNNs) combining those operations with cellular neural networks. So far researchers have founded that FCNNs are useful in image processing. Some results have been reported on dynamical behaviors including the existence and stability of equilibrium, periodic solution for FCNNs [14-18].

In contrast, however, very few results are available on the existence and exponential stability of anti-periodic solutions for fuzzy Cohen-Grossberg neural networks (FCGNNs).Arising from problems in applied sciences, the existence of anti-periodic solutions plays a key role in characterizing the behavior of nonlinear differential equations (see [19-24]). Moreover, both continuous and discrete systems are very important in implementing and applications. But it is troublesome to study the existence of anti-periodic solutions for discrete and continuous systems, respectively. Therefore it is meaningful to study FCGNNs on time scales, which was initiated by Hilger [25] in order to unify continuous and discrete systems.

Motivated by the above discussions, in this paper, we consider the following FCGNNs on time scales.

$$
\begin{aligned}
x_{i}^{\Delta}(t)= & -a_{i}\left(x_{i}(t)\right)\left[b_{i}\left(x_{i}(t)\right)-\sum_{j=1}^{n} c_{i j}(t) f_{j}\left(x_{j}(t)\right)-{ }_{j=1}^{n} \alpha_{i j}(t) g_{j}\left(x_{j}\left(t-\tau_{i j}(t)\right)\right)\right. \\
& \left.-{ }_{j=1}^{n} \beta_{i j}(t) g_{j}\left(x_{j}\left(t-\tau_{i j}(t)\right)\right)-I_{i}(t)\right]
\end{aligned}
$$


where $t \in \mathbb{T}, \mathbb{T}$ is an $\omega$-periodic time scale which has the subspace topology inherited from the standard topology on $\mathbb{R} . n$ corresponds to the number of units in the neural networks. For $i=1,2, \cdots, n, x_{i}(t)$ corresponds to the state of the $i$ th neuron. $f_{j}(\cdot), g_{j}(\cdot)$ are signal transmission functions. $\tau_{i j}(t)$ corresponds to the transmission delay along the axon of the $j$ th unit from the $i$ th unit and satisfies $0 \leq \tau_{i j}(t) \leq \tau$ ( $\tau$ is a constant). $a_{i}\left(x_{i}(t)\right)>0$ represents an amplification function at time $t . b_{i}\left(x_{i}(t)\right)$ is an appropriately behaved function at time $t ; c_{i j}(t)$ represents the elements of the feedback template. $I_{i}(t)$ is external input to the $i$ th unit. $\alpha_{i j}(t), \beta_{i j}(t)$ are elements of fuzzy feedback MIN template and fuzzy feedback MAX template, respectively; $\wedge$ and $\vee$ denote the fuzzy AND and fuzzy OR operation, respectively.

The main aim of this article is to establish some sufficient conditions for the existence and exponential stability of anti periodic solutions of (1). The organization of this paper is as follows. In Section 2, we introduce some definitions and lemmas. In Section 3, we establish sufficient conditions for the existence and exponential stability of the anti-periodic solutions of system (1). In Section 4, an example is given to demonstrate the effectiveness of our results. Conclusions are drawn in Section 5 .

\section{Preliminaries}

In this section, we shall first recall some basic definitions, lemmas which are used in what follows.

Let $\mathbb{T}$ be a nonempty closed subset (time scale) of $\mathbb{R}$. The forward and backward jump operators $\sigma, \rho: \mathbb{T} \rightarrow \mathbb{T}$ and the graininess $\mu: \mathbb{T} \rightarrow \mathbb{R}^{+}$are defined, respectively, by $\sigma(t)=\inf \{s \in \mathbb{T}: s>t\}$, $\rho(t)=\sup \{s \in \mathbb{T}: s<t\}, \mu(t)=\sigma(t)-t$. A point $t \in \mathbb{T}$ is called left-dense if $t>\inf \mathbb{T}$ and $\rho(t)=t$, left-scattered if $\rho(t)<t$, right-dense if $t<\sup \mathbb{T}$ and $\sigma(t)=t$, and right-scattered if $\sigma(t)>t$. If $\mathbb{T}$ has a left-scattered maximum $m$, then $\mathbb{T}^{k}=\mathbb{T} \backslash\{m\}$, otherwise $\mathbb{T}^{k}=\mathbb{T}$. If $\mathbb{T}$ has a right-scattered minimum $m$, then $\mathbb{T}_{k}=\mathbb{T} \backslash\{m\}$. otherwise $\mathbb{T}_{k}=\mathbb{T}$.

A function $f: \mathbb{T} \rightarrow R$ is right-dense continuous provided it is continuous at right-dense point in $\mathbb{T}$ and its left-side limits exist at left-dense points in $\mathbb{T}$. If $f$ is continuous at each right-dense point and each left-dense point, then $f$ is said to be a continuous function on $\mathbb{T}$.

For $y: \mathbb{T} \rightarrow \mathbb{R}$ and $t \in \mathbb{T}^{k}$, we define the delta derivative of $y(t), y^{\Delta}(t)$ to be the number (if exists) with the property that for given $\varepsilon>0$, there exists a neighborhood $U$ of $t$ such that $\left|[y(\sigma(t))-y(s)]-y^{\Delta}(t)[\sigma(t)-y(s)]\right|<\varepsilon|\sigma(t)-s|$ for all $s \in U$. If $y$ is continuous, then $y$ is rightdense continuous, and $y$ is delta differentiable at $t$, then $y$ is continuous at $t$. Let $y$ be right-dense continuous. If $Y^{\Delta}(t)=y(t)$, then we define the delta integral by $\int_{a}^{t} y(s) d s=Y(t)-Y(a)$

Definition 2.1 [27] If $a \in \mathbb{T}, \sup \mathbb{T}=\mathbb{R}$ and $f$ is rd-continuous on $[0, \infty)$, then we define the improper integral by $\int_{a}^{\infty} f(t) \Delta t=\lim _{b \rightarrow \infty} \int_{a}^{b} f(t) \Delta t$.

Provided this limit exists, and we say that the improper integral converges in this case. If this limit does not exist, then we say that the improper integral diverges.

Definition 2.2 [28] For each $t \in \mathbb{T}$, let $N$ be a neighborhood of $t$, then, for $V \in C_{r d}\left[\mathbb{T} \times \mathbb{R}^{n}, \mathbb{R}^{+}\right.$). Define $D^{+} V^{\Delta}(t, x(t))$ to mean that, given $\varepsilon>0$, there exists a right neighborhood $N_{\varepsilon} \subset N$ of $t$ such that

$$
\frac{V(\sigma(t, x(\sigma(t)))-V(s, x(\sigma(t)))-\mu(t, s) f(t, x(t))}{\mu(t, s)}<D^{+} V^{\Delta}(t, x(t))+\varepsilon
$$


for each $s \in N_{\varepsilon}, s>t$, where $\mu(t, s)=\sigma(t)-s$. If $t$ is $r d$ and $V(t, x(t))$ is continuous at $t$, this reduces to

$$
D^{+} V^{\Delta}(t, x(t))=\frac{V(\sigma(t), x(\sigma(t)))-V(t, x(\sigma(t)))}{\sigma(t)-t}
$$

Definition 2.3 [11] We say that a time scale $\mathbb{T}$ is periodic if there exists $p>0$ such that if $t \in \mathbb{T}$, then $t \pm p \in \mathbb{T}$. For $\mathbb{T} \neq \mathbb{R}$, the least positive $p$ is called the period of the time scale.

Let $\mathbb{T} \neq \mathbb{R}$ be a periodic time scale with periodic $p$. We say that the function $f: \mathbb{T} \rightarrow \mathbb{R}$ is $\omega$ anti-periodic if there exists a natural number $n$ such that $\omega=n p, f(t+\omega)=f(t)$ for all $t \in \mathbb{T}$ and $\omega$ is the least number such that $f(t+\omega)=f(t)$. If $\mathbb{T}=\mathbb{R}$, we say that $f$ is $\omega$ anti-periodic if $\omega$ is the least positive number such that $f(t+\omega)=f(t)$ for all $t \in \mathbb{T}$.

A function $r: \mathbb{T} \rightarrow R$ is called regressive if $1+\mu(t) r(t) \neq 0$, , for all $t \in \mathbb{T}^{k}$.

If $r$ is regressive function, then the generalized exponential function $e_{r}$ is defined by $e_{r}(t, s)=\exp \left\{\int_{s}^{t} \xi_{\mu}(r(\tau)) \Delta \tau\right\}, s, t \in \mathbb{T}$

with the cylinder transformation

$\xi_{h}(z)=\left\{\begin{array}{cc}\frac{\log (1+h z)}{h}, & h \neq 0 \\ z, & h=0\end{array}\right.$

Let $p, q: \mathbb{T} \rightarrow \mathbb{R}$ be two regressive functions, we define

$$
p \oplus q:=p+q+\mu p q ; p \Theta q:=p \oplus(\Theta q) ; \quad \Theta p:=\frac{p}{1+\mu p}
$$

Lemma 2.1. [12] Let $p, q$ be regressive functions on $\mathbb{T}$. Then

(i) $e_{0}(t, s)=1$ and $e_{p}(t, t)=1 ; \quad$ (ii) $e_{p}(\sigma(t), s)=(1+\mu(t) p(t)) e_{p}(t, s)$;

(iii) $e_{p}(t, s) e_{p}(s, r)=e_{p}(t, r) ; \quad$ (iv) $e_{p}^{\Delta}(\cdot, s)=p e_{p}(\cdot, s)$.

Lemma 2.2 [11] Assume that $f, g: \mathbb{T} \rightarrow \mathbb{R}$ are delta differentiable at $t \in \mathbb{T}^{k}$, then $(f g)^{\Delta}=f^{\Delta}(t) g(t)+f(\sigma(t)) g^{\Delta}(t)=f(t) g^{\Delta}(t)+f^{\Delta}(t) g(\sigma(t))$.

Lemma 2.3 [12] Assume that $p(t) \geq 0$, for $t>s$, then $e_{p}(t, s) \geq 1$.

Lemma 2.4 [11] Assume that $p \in \mathbb{R}$ is $\omega$ periodic, then $e_{p}(t+n \omega, s)=\left(e_{p}(t+\omega, s)\right)^{n}$ for $n \in \mathbb{N}$.

Lemma 2.5 [27] Let $f$ be continuous on $[a, b]_{\mathbb{T}}$ and delta differentiable on $[a, b)_{\mathbb{T}}$, then there exist $\xi, \varsigma \in[a, b)_{\mathbb{T}}$ such that $f^{\Delta}(\xi)(b-a) \leq f(b)-f(a) \leq f^{\Delta}(\varsigma)(b-a)$.

Lemma 2.6 [14] Suppose $x$ and $y$ are two states of system (1), then we have

$$
\left|\stackrel{n}{\wedge} \alpha_{i j}(t) g_{j}(x)-\bigwedge_{j=1}^{n} \alpha_{i j}(t) g_{j}(y)\right| \leq \sum_{j=1}^{n}\left|\alpha_{i j}(t) \| g_{j}(x)-g_{j}(y)\right|
$$

and

$$
\left|\stackrel{n}{\vee} \alpha_{i j}(t) g_{j}(x)-\vee_{j=1}^{n} \alpha_{i j}(t) g_{j}(y)\right| \leq \sum_{j=1}^{n}\left|\alpha_{i j}(t) \| g_{j}(x)-g_{j}(y)\right|
$$

Definition 2.4. The anti-periodic solution $x^{*}(t)=\left(x_{1}^{*}(t), x_{2}^{*}(t), \cdots, x_{n}^{*}(t)\right)^{T}$ of system (1) with initial value $\varphi^{*}(t)=\left(\varphi_{1}^{*}(t), \varphi_{2}^{*}(t), \cdots, \varphi_{n}^{*}(t)\right)^{T}$ is said to be globally exponentially stable if there exists a positive constant $M=M(\eta) \geq 1$ and $\varepsilon>0$ such that, for every $\eta \in \mathbb{T}$,

$$
\left\|x_{i}(t)-x_{i}^{*}\right\| \leq M e_{\theta \varepsilon}(t, \eta)\left\|\varphi-x^{*}\right\| .
$$


where $\left\|\varphi-x^{*}\right\|=\sup _{s \in[-\tau, 0]} \max _{t \in[0, \omega]_{\mathrm{T}}}\left|\varphi_{i}(s)-x_{i}^{*}(s)\right|$.

For the sake of convenience, we introduce some notations

$\tau=\max _{1 \leq i, j \leq n} \max _{t \in[0, \omega\}_{\mathbb{T}}}\left|\tau_{i j}(t)\right|, \bar{I}_{i}=\max _{t \in[0, \omega]_{\mathbb{T}}}\left|I_{i}(t)\right|, \quad \bar{c}_{i j}=\max _{t \in[0, \omega\}_{\mathbb{T}}}\left|c_{i j}(t)\right|, \bar{\alpha}_{i j}=\max _{t \in[0, \omega]_{\mathrm{T}}}\left|\alpha_{i j}(t)\right|, \bar{\beta}_{i j}=\max _{t \in[0, \omega\}_{\mathbb{T}}}\left|\beta_{i j}(t)\right|$.

Let $x(t)=\left(x_{1}(t), x_{2}(t), \cdots, x_{n}(t)\right)^{T} \in C\left(\mathbb{T}, \mathbb{R}^{n}\right),\|x\|=\sum_{i=1}^{n} \max _{t \in[0, \omega\}_{\mathbb{T}}}\left|x_{i}(t)\right|$

The initial conditions associated with system (1) are of the form

$$
x_{i}(t)=\varphi_{i}(t), t \in[-\tau, 0]_{\mathbb{T}},
$$

where $\varphi_{i}(t), i=1,2, \cdots, n$ are continuous functions on $[-\tau, 0]_{\mathbb{T}}$.

Let $x_{i}(t) \in C(\mathbb{T}, \mathbb{R}), x_{i}(t)$ is said to be $\omega$ anti periodic, if $x_{i}(t+\omega)=-x_{i}(t)$ for all $t \in \mathbb{T}, \omega>0$ is a constant. Denote $\mathbb{R}^{+}=[0,+\infty)$. Throughout this paper, we make the following assumptions

(A1) $c_{i j}, \alpha_{i j}, \beta_{i j}, I_{i} \in C(\mathbb{T}, \mathbb{R}), c_{i j}(t+\omega)=c_{i j}(t), \alpha_{i j}(t+\omega)=\alpha_{i j}(t), \beta_{i j}(t+\omega)=\beta_{i j}(t)$, $I_{i}(t+\omega)=-I_{i}(t)$, and $\tau_{i j} \in C(\mathbb{T}, \mathbb{T}), \tau_{i j}(t+\omega)=\tau_{i j}(t)$, where $\omega>0$ is a constant, $i, j=1,2, \cdots, n$.

(A2) $a_{i} \in C\left(\mathbb{R}, \mathbb{R}^{+}\right), a_{i}(-u)=a_{i}(u)$, and there exist positive constants $\bar{a}_{i}, \underline{a}_{i}$ such that $0 \leq \underline{a}_{i} \leq a_{i}(\cdot) \leq \bar{a}_{i}, i=1,2, \cdots, n$

(A3) $b_{i} \in C(\mathbb{R}, \mathbb{R}), b_{i}(-u)=-b_{i}(u)$ and there exist positive constants $\delta_{i}$ such that $\delta_{i}|u| \leq \operatorname{sign}(u) b_{i}(u)$ for all $u \in \mathbb{R}, i=1,2, \cdots, n$.

(A4) $f_{j}, g_{j} \in C\left(\mathbb{R} \mathbb{R}, f_{j}(-u)=-f_{j}(u), g_{j}(-u)=-g_{j}(u), f_{j}(0)=g_{j}(0)=0\right.$, and there exist $\mu_{j}, v_{j}(j=1,2, \cdots, n)$ such that

$\left|f_{j}(u)-f_{j}(v)\right| \leq \mu_{j}|u-v|,\left|g_{j}(u)-g_{j}(v)\right| \leq v_{j}|u-v|$.

for all $u, v \in \mathbb{R}, j=1,2, \cdots, n$.

\section{Main results}

In this section, we will prove our main results of this paper.

Lemma 3.1. Under condition (A1)-(A4), and suppose further the following condition hold

(A5) there exists a constant $\gamma>0$ such that

$$
-\underline{a}_{i} \delta_{i}+\bar{a}_{i} \sum_{j=1}^{n}\left[\bar{c}_{i j} \mu_{j}+\left(\overline{\alpha_{i j}}+\overline{\beta_{i j}}\right) v_{j}\right]<-\gamma<0 \text {. }
$$

Suppose that $\tilde{x}(t)=\left(\tilde{x}_{1}(t), \tilde{x}_{2}(t), \cdots, \tilde{x}_{n}(t)\right)^{T}$ is a solution of $(1)$ with initial condition

$$
\tilde{x}_{i}(s)=\tilde{\varphi}_{i}(s), \quad\left|\tilde{x}_{i}(s)\right|<\frac{\Gamma}{\gamma}, s \in[-\tau, 0], i=1,2, \cdots, n,
$$

where $\Gamma=\max _{1 \leq i \leq n}\left\{\bar{a}_{i} \bar{I}_{i}\right\}$. Then

$$
\left|\tilde{x}_{i}(t)\right|<\frac{\Gamma}{\gamma}, t \in \mathbb{T}^{+}, \quad i=1,2, \cdots, n .
$$

Proof. For any given initial condition, assumption (A4) guarantees the existence and unique of $x(t)$, the solution to (1) in $[-\tau,+\infty)_{\mathbb{T}}$. By way of contradiction, assume that (4) does not hold. Then, there exist $i \in\{1,2, \cdots, n\}$, and $t_{0} \in \mathbb{T}^{+}$such that

$$
\left|\tilde{x}_{i}\left(t_{0}\right)\right|=\frac{\Gamma}{\gamma},\left|\tilde{x}_{i}(t)\right|<\frac{\Gamma}{\gamma}, \quad t \in\left[-\tau, t_{0}\right)_{\mathbb{T}} \text {, and }\left|\tilde{x}_{j}(t)\right|<\frac{\Gamma}{\gamma}, t \in\left[-\tau, t_{0}\right)_{\mathbb{T}}, j \neq i, j=1,2, \cdots, n .
$$

By directly computing the upper left derivative of $x_{i}(t)$, Combining with (A1)-(A5), we can obtain that 


$$
\begin{aligned}
& 0 \leq D^{+}\left|\tilde{x}_{i}\left(t_{0}\right)\right|^{\Delta} \leq \operatorname{sign}\left(\tilde{x}_{i}\left(t_{0}\right)\right)\left\{-a_{i}\left(\tilde{x}_{i}\left(t_{0}\right)\right)\left[b_{i}\left(\tilde{x}_{i}\left(t_{0}\right)\right)-\sum_{j=1}^{n} c_{i j}(t) f_{j}\left(\tilde{x}_{j}\left(t_{0}\right)\right)\right.\right. \\
& \left.\left.-\stackrel{n}{j=1}^{n} \alpha_{i j}(t) g_{j}\left(\tilde{x}_{i}\left(t_{0}-\tau_{i j}\left(t_{0}\right)\right)\right)-\vee_{j=1}^{n} \beta_{i j}(t) g_{j}\left(\tilde{x}_{i}\left(t_{0}-\tau_{i j}\left(t_{0}\right)\right)\right)-I_{i}\left(t_{0}\right)\right]\right\} \\
& \leq-a_{i}\left(\tilde{x}_{i}\left(t_{0}\right)\right) \operatorname{sign}\left(\tilde{x}_{i}\left(t_{0}\right)\right) b_{i}\left(\tilde{x}_{i}\left(t_{0}\right)\right)+\mid a_{i}\left(\tilde{x}_{i}\left(t_{0}\right)\right) \sum_{j=1}^{n} c_{i j}(t) f_{j}\left(\tilde{x}_{j}\left(t_{0}\right)\right) \\
& +a_{i}\left(\tilde{x}_{i}\left(t_{0}\right)\right) \stackrel{n}{\wedge} \alpha_{i j}(t) g_{j}\left(\tilde{x}_{i}\left(t_{0}-\tau_{i j}\left(t_{0}\right)\right)\right)+a_{i}\left(\tilde{x}_{i}\left(t_{0}\right)\right) \underset{j=1}{\stackrel{n}{\vee}} \beta_{i j}(t) g_{j}\left(\tilde{x}_{i}\left(t_{0}-\tau_{i j}\left(t_{0}\right)\right)\right)+a_{i}\left(\tilde{x}_{i}\left(t_{0}\right)\right) I_{i}\left(t_{0}\right) \mid \\
& \leq-a_{i}\left(\tilde{x}_{i}\left(t_{0}\right)\right) \operatorname{sign}\left(\tilde{x}_{i}\left(t_{0}\right)\right) b_{i}\left(\tilde{x}_{i}\left(t_{0}\right)\right)+a_{i}\left(\tilde{x}_{i}\left(t_{0}\right)\right)\left|\sum_{j=1}^{n} c_{i j}(t) f_{j}\left(\tilde{x}_{j}\left(t_{0}\right)\right)-\sum_{j=1}^{n} c_{i j}(t) f_{j}(0)\right| \\
& +a_{i}\left(\tilde{x}_{i}\left(t_{0}\right)\right)\left|\bigwedge_{j=1}^{n} \alpha_{i j}(t) g_{j}\left(\tilde{x}_{i}\left(t_{0}-\tau_{i j}\left(t_{0}\right)\right)\right)-\hat{j}_{j=1}^{n} \alpha_{i j}(t) g_{j}(0)\right| \\
& +a_{i}\left(\tilde{x}_{i}\left(t_{0}\right)\right)\left|\underset{j=1}{\vee} \beta_{i j}(t) g_{j}\left(\tilde{x}_{i}\left(t_{0}-\tau_{i j}\left(t_{0}\right)\right)\right)-{\underset{j=1}{\vee}}_{i j}^{n} \beta_{i j}(t) g_{j}(0)\right|+\left|a_{i}\left(\tilde{x}_{i}\left(t_{0}\right)\right) I_{i}\left(t_{0}\right)\right| \\
& \leq-\underline{a}_{i} \delta_{i}\left|\tilde{x}_{i}\left(t_{0}\right)\right|+\bar{a}_{i} \sum_{j=1}^{n} \bar{c}_{i j} \mu_{j}\left|\tilde{x}_{j}\left(t_{0}\right)\right|+\bar{a}_{i} \sum_{j=1}^{n}\left(\bar{\alpha}_{i j}+\overline{\beta_{i j}}\right) v_{j}\left|\tilde{x}_{j}\left(t_{0}-\tau_{i j}\left(t_{0}\right)\right)\right|+\bar{a}_{i} \bar{I}_{i} \\
& \left.\leq\left[-\underline{a}_{i} \delta_{i}+\bar{a}_{i} \sum_{j=1}^{n} \bar{c}_{i j} \mu_{j}+\left(\bar{\alpha}_{i j}+\bar{\beta}_{i j}\right) v_{j}\right)\right] \frac{\Gamma}{\gamma}+\bar{a}_{i} \bar{I}_{i} \\
& \leq-\gamma \frac{\Gamma}{\gamma}+\bar{a}_{i} \bar{I}_{i} \leq 0
\end{aligned}
$$

(A6) There exists a positive constant $\rho_{i}$ such that for all $u, v \in R$ $\left|a_{i}(u)-a_{i}(v)\right| \leq \rho_{i}|u-v|, i=1,2, \cdots, n$.

(A7) There exists a positive constant $\xi_{i}$ such that for all $u, v \in R$,

$\left[a_{i}(u) b_{i}(u)-a_{i}(v) b_{i}(v)\right](u-v) \geq 0,\left|a_{i}(u) b_{i}(u)-a_{i}(v) b_{i}(v)\right| \geq \xi_{i}|u-v|, i=1,2, \cdots, n$,

Lemma 3.2 Let (A1)-(A7) hold, and let $x^{*}(t)=\left(x_{1}^{*}(t), x_{2}^{*}(t), \cdots, x_{n}^{*}(t)\right)^{T}$ be the solution of (1) with initial conditions (3), suppose that

$$
--\xi_{i}+\rho_{i} \sum_{j=1}^{n}\left(\overline{c_{i j}}+\overline{\alpha_{i j}}+\overline{\beta_{i j}}\right) \frac{\Gamma}{\gamma}+\rho_{i} \bar{I}_{i}+\bar{a}_{i} \sum_{j=1}^{n}\left(\overline{c_{i j}} \mu_{j}+\left(\overline{\alpha_{i j}}+\overline{\beta_{i j}}\right) \nu_{j}\right)<0, i=1,2, \cdots, n,
$$

Then $x^{*}(t)$ is exponentially stable.

Proof. The proof is similar to Lemma 3.2 of [22]. So we omit it.

Theorem 3.1. Suppose (A1)-(A8) hold, then (1) has an anti periodic solution $x^{*}(t)$ which is globally exponentially stable.

Proof. Let $x(t)=\left(x_{1}(t), x_{2}(t), \cdots, x_{n}(t)\right)^{T}$ be a solution of (1) with initial condition

$$
x_{i}(s)=\varphi_{i}(s),\left|\varphi_{i}(s)\right|<\frac{\Gamma}{\gamma}, s \in[-\tau, 0]_{T}, i=1,2, \cdots, n \text {. }
$$

By Lemma 3.1, the solution $x(t)$ is bounded and

$$
\left|x_{i}(t)\right|<\frac{\Gamma}{\gamma}, t \in[-\tau, \infty]_{T}, i=1,2, \cdots, n \text {. }
$$

From (1), (A1)-(A4) and Lemma 2.1, we have

$$
\begin{aligned}
& \left|(-1)^{k+1} x_{i}(t+(k+1) \omega)\right|^{\Delta}=(-1)^{k+1} x_{1}^{\Delta}(t+(k+1) \omega) \\
& =(-1)^{k+1}\left\{-a_{i}\left(x_{i}(t+(k+1) \omega)\right)\left[b_{i}\left(x_{i}(t+(k+1) \omega)\right)-\sum_{j=1}^{n} c_{i j}(t+(k+1) \omega) f_{j}\left(x_{j}(t+(k+1) \omega)\right)\right.\right.
\end{aligned}
$$




$$
\begin{aligned}
& -\hat{j}_{j=1}^{n} \alpha_{i j}(t+(k+1) \omega) g_{j}\left(x_{j}\left(t+(k+1) \omega-\tau_{i j}(t+(k+1) \omega)\right)\right) \\
& \left.\left.-\underset{j=1}{\vee} \beta_{i j}(t+(k+1) \omega) g_{j}\left(x_{j}\left(t+(k+1) \omega-\tau_{i j}(t+(k+1) \omega)\right)\right)-I_{i}(t+(k+1) \omega)\right]\right\} \\
& =-a_{i}\left((-1)^{k+1} x_{i}(t+(k+1) \omega)\right)\left[b_{i}\left((-1)^{k+1} x_{i}(t+(k+1) \omega)\right)\right. \\
& -\sum_{j=1}^{n} c_{i j}(t) f_{j}\left((-1)^{k+1} x_{j}(t+(k+1) \omega)-\hat{\jmath}_{j=1}^{n} \alpha_{i j}(t) g_{j}\left(x_{j}\left(t+(k+1) \omega-\tau_{i j}(t)\right)\right)\right. \\
& \left.-\stackrel{n}{\underset{j}{\vee} \beta_{i j}} \beta_{i j}(t) g_{j}\left(x_{j}\left(t+(k+1) \omega-\tau_{i j}(t)\right)\right)-I_{i}(t)\right]
\end{aligned}
$$

Therefore, for any natural number $k,(-1)^{k+1} x_{j}(t+(k+1) \omega)$ is the solution of (1). Then, from Lemma 3.2, there exists a constant $M>1$, such that

$$
\begin{aligned}
\left|(-1)^{k+1} x_{i}(t+(k+1) \omega)-(-1)^{k} x_{i}(t+k \omega)\right| \leq & M e_{\theta \varepsilon}(t+k \omega, \eta) \sup _{s \in[-\tau, 0]} \max _{1 \leq i \leq n}\left|x_{i}(s+\omega)-x_{i}(s)\right| \\
& \leq 2 M e_{\theta \varepsilon}(t+k \omega, \eta) \frac{\Gamma}{\gamma}
\end{aligned}
$$

where $t+k \omega \in T^{+}, i=1,2, \cdots, n$. Thus, for any natural $p$, we have

$$
(-1)^{p+1} x_{i}(t+(p+1) \omega)=x_{i}(t)+\sum_{k=0}^{p}\left[(-1)^{k+1} x_{i}(t+(k+1) \omega)-(-1)^{k} x_{i}(t+k \omega)\right]
$$

Then

$$
\left|(-1)^{p+1} x_{i}(t+(p+1) \omega)\right|=\left|x_{i}(t)\right|+\sum_{k=0}^{p}\left|(-1)^{k+1} x_{i}(t+(k+1) \omega)-(-1)^{k} x_{i}(t+k \omega)\right|
$$

In view of (6), we can choose a sufficiently large constant $N>0$ and a positive constant $\varsigma$ such that

$$
\left|(-1)^{k+1} x_{i}(t+(k+1) \omega)-(-1)^{k} x_{i}(t+k \omega)\right|<\varsigma\left(e_{\theta \varepsilon}(t+\omega, \eta)\right)^{K}, k>N .
$$

On any compact set of $R$. Together with (7) and (8), it follows that $\left\{(-1)^{p} x_{i}(t+p \omega)\right\}$ uniformly converges to a continuous function $x^{*}(t)$ on any compact set of $R$.

Now, we need to prove that $x^{*}(t)$ is an anti periodic solution of (1). First $x_{i}^{*}(t)$ is $\omega$ anti-periodic, since

$$
x_{i}^{*}(t+\omega)=\lim _{p \rightarrow \infty}(-1)^{p} x_{i}(t+p \omega)=-\lim _{p \rightarrow \infty}(-1)^{p+1} x_{i}(t+(p+1) \omega)=-x_{i}^{*}(t)
$$

Next, we show that $x^{*}(t)$ is a solution of (1). Noting (1) and (5), we obtain that $\left\{\left((-1)^{p+1} x_{i}(t+(p+1) \omega)\right)^{\Delta}\right\}$ uniformly converges to continuous function on any compact set of $R$. letting $p \rightarrow \infty$, we have

$$
\begin{aligned}
& \left(x_{i}^{*}(t)\right)^{\Delta}=-a_{i}\left(x_{i}^{*}(t)\right)\left[b_{i}\left(x_{i}^{*}(t)\right)-\sum_{j=1}^{n} c_{i j}(t) f_{j}\left(x_{j}^{*}(t)\right)-\hat{j}_{j=1}^{n} \alpha_{i j}(t) g_{j}\left(x_{j}^{*}\left(t-\tau_{i j}(t)\right)\right)\right. \\
& \left.-\vee_{j=1}^{n} \beta_{i j}(t) g_{j}\left(x_{j}^{*}\left(t-\tau_{i j}(t)\right)\right)-I_{i}(t)\right]
\end{aligned}
$$

That is $x^{*}(t)$ is a solution of (1). It follows from Lemma 3.2 that $x^{*}(t)$ is exponentially stable. This completes the proof of Theorem 3.1.

\section{An illustrative example}

In this section, we will give an example to illustrate the feasibility and effectiveness of our results obtained in Section 3. delays

Example 4.1 Consider the following fuzzy Cohen-Grossberg neural networks with time-varying 


$$
\begin{aligned}
x_{i}^{\Delta}(t)=-a_{i}\left(x_{i}(t)\right) & {\left[b_{i}\left(x_{i}(t)\right)-\sum_{j=1}^{2} c_{i j}(t) f_{j}\left(x_{j}(t)\right)-\underset{j=1}{2} \alpha_{i j}(t) g_{j}\left(x_{j}\left(t-\tau_{i j}(t)\right)\right)\right.} \\
& \left.-\underset{j=1}{\vee} \beta_{i j}(t) g_{j}\left(x_{j}\left(t-\tau_{i j}(t)\right)\right)-I_{i}(t)\right], \quad t \in T^{+},
\end{aligned}
$$

where

$a_{1}(u)=5+\cos u, a_{2}(u)=5-\cos u, b_{1}(u)=b_{2}(u)=u, f_{j}(u)=g_{j}(u)=\sin u$,

$(i, j=1,2)$.

$$
\begin{gathered}
\left(c_{i j}\right)_{2 \times 2}=\left(\begin{array}{cc}
0.09|\sin (4 \pi t)| & 0.07|\cos (4 \pi t)| \\
0.06|\cos (4 \pi t)| & 0.08|\sin (4 \pi t)|
\end{array}\right), \quad\left(\alpha_{i j}\right)_{2 \times 2}=\left(\begin{array}{cc}
0.16|\cos (4 \pi t)| & 0.14|\sin (4 \pi t)| \\
0.12|\sin (4 \pi t)| & 0.08|\cos (4 \pi t)|
\end{array}\right), \\
\left(\beta_{i j}\right)_{2 \times 2}=\left(\begin{array}{ll}
0.05|\sin (4 \pi t)| & 0.04|\cos (4 \pi t)| \\
0.06|\cos (4 \pi t)| & 0.06|\sin (4 \pi t)|
\end{array}\right), \quad\left(I_{i}\right)_{2 \times 1}=\left(\begin{array}{c}
0.2 \sin (4 \pi t) \\
0.1 \cos (4 \pi t)
\end{array}\right),
\end{gathered}
$$

then, we have

$\overline{a_{1}}=\overline{a_{2}}=6, \underline{a_{1}}=\underline{a_{2}}=4, \overline{c_{11}}=0.09, \overline{c_{22}}=0.08, \overline{c_{12}}=0.07, \overline{c_{21}}=0.06, \overline{\alpha_{11}}=0.16, \overline{\alpha_{22}}=0.08$,

$\overline{\alpha_{12}}=0.14, \overline{\alpha_{21}}=0.12, \overline{\beta_{11}}=0.05, \overline{\beta_{22}}=0.06, \overline{\beta_{12}}=0.04, \overline{\beta_{21}}=0.06, \overline{I_{1}}=0.2, \overline{I_{2}}=0.1$,

$\rho_{1}=\rho_{2}=1, \delta_{1}=\delta_{2}=1, \xi_{1}=\xi_{2}=4, \Gamma=2,4, \mu_{j}=v_{j}=1(j=1,2)$

Take $\gamma=0.6$, By simple computation, we have

$$
\begin{aligned}
& -\underline{a_{1}} \delta_{1}+\bar{a}_{1} \sum_{j=1}^{2}\left[\overline{c_{1 j}} \mu_{j}+\left(\overline{\alpha_{1 j}}+\overline{\beta_{1 j}}\right) v_{j}\right]=-0.7<0,-\underline{a_{2}} \delta_{2}+\overline{a_{2}} \sum_{j=1}^{2}\left[\overline{c_{2 j}} \mu_{j}+\left(\overline{\alpha_{2 j}}+\overline{\beta_{2 j}}\right) v_{j}\right]=-1.24<0, \\
& -\xi_{1}+\rho_{1} \sum_{j=1}^{2}\left(\overline{c_{1 j}}+\overline{\alpha_{1 j}}+\overline{\beta_{1 j}}\right) \frac{\Gamma}{\gamma}+\rho_{1} \overline{I_{1}}+\overline{a_{1}} \sum_{j=1}^{2}\left[\overline{c_{1 j}} \mu_{j}+\left(\overline{\alpha_{1 j}}+\overline{\beta_{1 j}}\right) v_{j}\right)=-1.2<0, \\
& -\xi_{2}+\rho_{2} \sum_{j=1}^{2}\left(\overline{c_{2 j}}+\overline{\alpha_{2 j}}+\overline{\beta_{2 j}}\right) \frac{\Gamma}{\gamma}+\rho_{2} \overline{I_{2}}+\overline{a_{2}} \sum_{j=1}^{2}\left[\overline{c_{2 j}} \mu_{j}+\left(\overline{\alpha_{2 j}}+\overline{\beta_{2 j}}\right) \nu_{j}\right)=-2.06<0 .
\end{aligned}
$$

Now, we can see that conditions (A1)-(A8) hold. By Theorem 3.1, system (9) has a $\frac{1}{4}$-anti-periodic solution which is exponentially stable.

\section{Conclusion}

In this paper, we have studied the existence, exponential stability of the anti-periodic solution for fuzzy Cohen-Grossberg neural networks with time delays. Some sufficient conditions set up here are easily verified and these conditions are correlated with parameters of the system (1). The obtained criteria can be applied to design globally exponential stable of anti-periodic fuzzy Cohen-Grossberg neural networks.

\section{Acknowledgements}

This work is supported by the National Natural Science Foundation of China (Grant No. 11361012).

\section{References}

[1] Cohen, M.and Grossberg,S. (1983) Stability and global pattern formation and memory storage by competitive neural networks. IEEE Trans Syst, Man Cyber 13, 815-826.

[2] Liang, J. and Cao,J. (2007) Global output convergence of recurrent neural networks with distributed delays, Nonlinear Analysis RWA 8, 187-197. 
[3] Chen,X. and Song,Q. (2010) Global exponential stability of the periodic solution of delayed Cohen-Grossberg neural networks with discontinuous activations, Neurocomputing, 73(16-18), 3097-3104.

[4] Li, C.and Yang, S.(2009) Existence and attractivity of periodic solutions to non-autonomous Cohen-Grossberg neural networks with time delays, Chaos, Solitons and Fractals, 41, 1235$1244 .$.

[6] Arik, S. and Orman, Z.(2005) Global stability analysis of Cohen-Grossberg neural networks with time varying delays, Physics Letters A 341, 410-421.

[7] Song, Q. and Cao,J.(2006) Stability analysis of Cohen-Grossberg neural network with both timevarying and continuously distributed delays, Journal of Computational and Applied Mathematics 197, 188-203.

[8] Bai, C. (2008) Stability analysis of Cohen-Grossberg BAM neural networks with delays and impulses, Chaos, Solitons and Fractals 35, 263-267

[9] Song, Q. and Zhang, J.(2008) Global exponential stability of impulsive Cohen-Grossberg neural network with time-varying delays, Nonlinear Analysis RWA 9, 500-510.

[9] Li, Y. , Chen, X. and Zhao, L. (2009) Stability and existence of periodic solutions to delayed Cohen-Grossberg BAM neural networks with impulses on time scales. Neurocomputing 72, $1621-1630$

[10] Li,Y., Zhao, L. and Zhang,T. (2011) Global exponential stability and existence of periodic solution of impulsive Cohen-Grossberg neural networks with distributed delays on time scales, Neural Process Lett 33, 61-81.

[11] Bohner, M. and Peterson , A. (2001) Dynamic equations on time scales, an introducation with applications.

Birkhauser, Boston.

[12] Bohner, M. and Peterson, A. (2003) Advances in dynamic equations on time scales. Birkhauser, Boston.

[13] Yang, T. and Yang, L.B.(1996) The global stability of fuzzy cellular neuralnetworks.IEEE Trans. Circ. Syst. I 43, 880--883.

[14] Yang, T., Yang,L.,Wu, C. and Chua, L. (1996) Fuzzy cellular neural networks: theory. Proc IEEE Int Workshop Cellular Neural Networks Appl., 181--186.

[15] Yang, T., Yang,L.,Wu, C. and Chua, L. (1996) Fuzzy cellular neural networks: applications, In Pro. of IEEE Int. Workshop on Cellular Neural Neworks Appl. 225--230.

[16] Huang, T.(2007) Exponential stability of delayed fuzzy cellular neural networks with diffusion, Chaos Solitons Fractals 31, 658-664.

[17] Zhang, Q. and Xiang,R.(2008) Global asymptotic stability of fuzzy cellular neural networks with time-varying delays, Phy. Lett. A 372, 3971--3977.

[18] Yuan,K. Cao,J. and Deng, J. (2006) Exponential stability and periodic solutions of fuzzy cellular neural networks with time-varying delays, Neurocomputing 69 , 1619-1627.

[19] Hu, M. and Wang, L.(2010) Existence and stability of anti-periodic solutions for an impulsive Cohen-Grossberg SICNNs on time scales, International Journal of Mathematical and Computer Sciences 6, 159-165.

[20] Shao, J. Y. (2009) An anti-periodic solution for a class of recurrent neural networks, J. Comput. Appl. Math. 228, 231-237.

[21] Shao, J.Y. (2008) Anti-periodic solutions for shunting inhibitory cellular neural networks with time-varying delays, Phys. Lett. A 372, 5011-5016.

[22] Li, Y. K.and Yang, L.(2009) Anti-periodic solutions for Cohen-Grossberg neural networks with bounded and unbounded delays, Commun. Nonlinear Sci.Numer. Simulat. 14, 3134-3140.

[23] Peng, G. Q.and Huang,L.H. (2009) Anti-periodic solutions for shunting inhibitory cellular neural networks with continuously distributed delays, Nonlinear Anal.: Real World Appl. 10 , 2434-2440.

[24] Fan, Q.Y. ,Wang, W.T. and Yi, X.J. (2009) Anti-periodic solutions for a class of $n$ th-order differential equations with delay, J. Comput. Appl. Math.230, 762-769. 
[25]Hilger, S. (1990) Analysis on measure chains--a unifiedapproach to continuous and discrete calculus, Results in Mathematics, , 18, 18-56.

[26] Bi, L , Bohner, M. and Fan, M.(2008) Periodic solutions of functional dynamic equations with infinite delay.

Nonlinear Anal 68, 1226-1245.

[27] Guseinov, G. (2003) Integration on time scales, J. Math. Anal. Appl. 285,107-127.

[28] Kaufmann, E. R. and Raffoul,Y. N.(2006) Periodic solutions for a neutral nonlinear dynamic equation on a time scale. J Math Anal Appl 319, 315-325. 